\title{
Dementia and out-of-pocket spending on health care services
}

\author{
Adeline Delavande $^{\mathrm{a}, \mathrm{b}}$, Michael D. Hurd ${ }^{\mathrm{c}}$, Paco Martorell ${ }^{\mathrm{b}, *}$, Kenneth M. Langa ${ }^{\mathrm{d}, \mathrm{e}}$ \\ ${ }^{a}$ University of Essex, Institute for Social and Economic Research, Colchester, Essex, United Kingdom \\ ${ }^{b}$ RAND Corporation, Santa Monica, CA, USA \\ ${ }^{c}$ RAND Corporation, NBER, NETSPAR and MEA, Santa Monica, CA, USA \\ ${ }^{d}$ Department of Medicine and Institute for Social Research, University of Michigan, Ann Arbor, MI, USA \\ ${ }^{e}$ Veterans Affairs Center for Clinical Management Research, Ann Arbor, MI, USA
}

\begin{abstract}
Background: High levels of out-of-pocket (OOP) spending for health care may lead patients to forego needed services and medications as well as hamper their ability to pay for other essential goods. Because it leads to disability and the loss of independence, dementia may put patients and their families at risk for high OOP spending, especially for long-term care services.

Methods: We used data from the Aging, Demographics, and Memory Study, a nationally representative subsample $(n=743)$ of the Health and Retirement Study, to determine whether individuals with dementia had higher self-reported OOP spending compared with those with cognitive impairment without dementia and those with normal cognitive function. We also examined the relationship between dementia and utilization of dental care and prescription medications - two types of health care that are frequently paid for OOP. Multivariate and logistic regression models were used to adjust for the influence of potential confounders.

Results: After controlling for demographics and comorbidities, those with dementia had more than three times the yearly OOP spending compared with those with normal cognition $(\$ 8216$ for those with dementia vs. $\$ 2570$ for those with normal cognition, $P<.01)$. Higher OOP spending for those with dementia was mainly driven by greater expenditures on nursing home care $(P<.01)$. Dementia was not associated with the likelihood of visiting the dentist $(P=.76)$ or foregoing prescription medications owing to cost $(P=.34)$.

Conclusions: Dementia is associated with high levels of OOP spending but not with the use of dental care or foregoing prescription medications, suggesting that excess OOP spending among those with dementia does not "crowd out" spending on these other health care services.
\end{abstract}

(C) 2013 The Alzheimer's Association. All rights reserved.

Keywords: Dementia; CIND; Out-of-pocket spending; Nursing home care; Population-based study

\section{Introduction}

The Medicare health insurance program offers nearly universal coverage for Americans aged 65 years and older, but paying for deductibles, copayments, and uncovered services may, nonetheless, represent a substantial financial burden. High levels of out-of-pocket (OOP) spending for health care are potentially problematic because they may lead patients to forego needed services and medications as well as hamper their ability to pay for other essential goods, such as food and housing, leading to sharp reductions in

*Corresponding author. Tel.: 310-393-0411; Fax: 310-260-8011.

E-mail address: paco@rand.org quality of life [1-4]. High OOP spending among those with illness and injury has also been cited as a common cause for personal bankruptcy [5] The significance of this issue is underscored by recent trends toward increasing OOP health care spending; 1 in 10 Medicare recipients spend at least $60 \%$ of their income on health care costs, up from $48 \%$ in 1997 [6]. Concerns about OOP spending have risen to prominence in the national policy discussion. Medicare Part D was enacted in 2006 mainly to protect seniors from high OOP spending on prescription drugs [7]. There are also calls to extend Medicare coverage to long-term care, another current source of high OOP expenditures [8].

Dementia is a chronic condition of aging characterized by cognitive decline that leads to a loss of independence. Owing 
to population aging, our calculations based on age-specific rates of dementia and population projections suggest that by 2040 the number of dementia cases will nearly triple (from approximately 3.4 million to 9.5 million cases). Because dementia limits the ability to function independently, demented individuals are much more likely to use long-term care facilities, such as nursing homes [9-11]. Medicare and private insurance coverage for these services are limited, and therefore, spending on long-term care represents the largest share of OOP expenditures for Medicare recipients [12]. Medicaid, the federal-state insurance program, does provide coverage for long-term care, but patients typically must meet income and asset tests to be eligible for Medicaid (eligibility rules vary across states). Consequently, patients suffering from dementia are at high risk of incurring very large OOP expenditures until they have "spent down" their assets below the Medicaid eligibility threshold [13-15].

Although there are reasons to suspect that dementia leads to higher levels of spending and health care utilization, the evidence remains mixed. Some studies find higher levels of cost and utilization among demented individuals [16-19], whereas others find reductions $[20,21]$. A key limitation of this line of research is the lack of data with reliable information on both costs and dementia diagnoses. Studies [17] using claims records have high-quality data on costs incurred by the payer, but they severely understate the prevalence of dementia and have limited information on spending on uncovered services (such as long-term care) [11,22-24]. Other studies use self-reported levels of OOP spending available in nationally representative data such as the Health and Retirement Study (HRS), but until recently, these data had no information on actual diagnoses of dementia $[19,21]$. In addition, owing to the sampling frame of the HRS, the samples used in earlier analyses likely undersampled individuals residing in nursing homes, who are precisely those at the greatest risk for very large OOP expenses [19]. Finally, no studies we are aware of have directly examined whether dementia reduces spending on types of medical care that are not directly related to dementia and that are typically paid for OOP. Were that to be the case, a possible explanation would be that high spending for dementia reduces economic resources for other types of health care spending, which could have additional health impacts.

This article addresses these limitations using newly available data from the Aging, Demographics, and Memory Study (ADAMS), a substudy of the HRS. The ADAMS includes clinical diagnoses of dementia status for a sample of individuals drawn from the HRS. We estimate the relationship between dementia and OOP spending for health care as well as between dementia and two types of health care that are typically paid for OOP and are not associated with dementia. Specifically, we use these data to test the following three hypotheses:
1. Dementia is associated with higher average OOP spending.

2. The increase in average OOP spending is driven by the incidence of large OOP expenditures on longterm care services, which are heavily used by individuals with dementia but are not typically covered by insurance.

3. Dementia reduces utilization of two types of health care (dental care and prescription drugs) that are not directly associated with care for dementia and that are typically paid for OOP.

This study is the first of its kind to use nationally representative data that combine information on (1) clinical diagnoses of dementia, (2) information on health care utilization and expenditures, and (3) detailed information on demographic characteristics and comorbidities that may confound the relationship between dementia and OOP spending. It is also the first study that we are aware of that uses nationally representative data to explicitly address whether dementia reduces utilization of two types of health care that are usually paid for OOP.

\section{Data}

\subsection{HRS and ADAMS}

This study examines the relationship between OOP spending reported in the HRS and diagnoses of dementia status made among individuals in the ADAMS. The HRS is a nationally representative longitudinal survey of individuals aged 51 years and older that began in 1992. Since 1996, the HRS core interviews have been conducted every 2 years. All individuals who enter the HRS are recruited from the community, but the HRS follows subjects even if they enter facilities such as nursing homes. The core interviews collect information on a wide variety of topics, including health status, health care utilization and spending, wealth and income, and employment histories [25]. All spouses of HRS respondents are interviewed, regardless of year of birth. Proxy interviews are used for study subjects who are unable to complete the survey interview without assistance.

HRS respondents aged 70 years and older formed the basis for the ADAMS sampling frame, the first nationally representative study of dementia in the United States. Subjects in ADAMS were drawn from either the 2000 or 2002 HRS. Sampling was stratified based on age, gender, and cognitive functioning, as measured by the cognitive assessment administered to HRS respondents. The final ADAMS sample consists of 856 respondents of 1770 who were recruited, which gives a response rate of $56 \%$, net of mortality [26]. We further limit the sample to the 743 individuals who completed the HRS interview after the ADAMS assessment because, as explained later, this was when information on health care expenditures made during the period of the ADAMS assessment was collected. 
An important advantage of the ADAMS sample is that it includes a more representative sample of individuals living in nursing homes than was available in earlier studies. Because the HRS initially interviews only those individuals residing in the community, early waves of the HRS contained relatively few nursing home residents. Consequently, the relationship between health care utilization and dementia may have been understated in earlier research that used data from the 1995 wave of the HRS [19,21]. In contrast, the ADAMS has more complete data on individuals residing in nursing homes, the population most at risk for large OOP expenditures owing to dementia.

\subsection{Dementia status}

Subjects recruited to be in the ADAMS and who agreed to participate in the study received an in-home clinical assessment of dementia status between the core HRS waves. The procedures underlying the diagnosis are described in detail in the article by Langa et al [27]. Briefly, these diagnoses were based on data collected during a 3- to 4-hour evaluation conducted approximately 1 year after the most recent HRS interview. Information was collected from a knowledgeable informant, from the respondent, and from medical records. A panel of experts (psychologists, psychiatrists, neurologists, and internists) then reviewed this information and assigned diagnoses, which fell within three general categories- "normal," "cognitive impairment, not demented" (CIND), or "demented." In our analysis sample, $11.4 \%$ of the subjects were diagnosed as demented, $21.4 \%$ as CIND, and $67.2 \%$ as normal, where these are weighted averages to account for the stratified sampling in ADAMS.

\subsection{OOP spending and health care utilization measures}

In the core interview, HRS respondents (or proxy respondents) are asked about their utilization and health care coverage, and then whether they incurred any OOP expenses. Starting with the 2002 wave, the HRS asked about spending on the following services: (1) nursing home stays, (2) hospital stays, (3) doctor visits, (4) dental visits, (5) outpatient surgery, (6) home health care, (7) other "special" services, (8) prescription drugs, and (9) dental services. Our analyses examine annual spending. For all spending aside from that on prescription drugs, the questions refer to total OOP spending incurred over the past 2 years, which we convert to annual values by dividing the reported amounts by 2 . The questions about spending on prescription drugs ask about average monthly OOP spending over the past 2 years, which is converted to an annual total by multiplying by 12 . All values are converted to 2010 dollars using the medical care consumer price index.

We analyze total annual OOP spending as well as OOP spending by health care type. Specifically, we separate OOP expenditures into those for nursing home stays, spending on prescription drugs, and spending on all other types of medical care. Total spending is partitioned in this way for two reasons. First, public and private insurance coverage for nursing home care and prescription drugs is typically much less generous than it is for other types of care. Second, existing evidence suggests that dementia increases the likelihood of nursing home stays [9].

Because long nursing home stays owing to dementia could result in extremely large OOP spending costs, the second type of outcome we use is an indicator variable for the incidence of very high annual OOP spending, which is defined as spending greater than $\$ 5000$ per year (roughly the 85th percentile of the OOP spending distribution, and $23 \%$ of median income in the sample). Other cutoff values were explored as sensitivity analyses, which are discussed later in the text.

A key issue in this study is the quality of the self-reported information on OOP spending, which, as previous research suggests, is often understated [28,29]. However, underreporting is mitigated in the HRS questionnaire in two ways. First, separate questions are asked about spending on various services, which helps respondents recall incidents that may be forgotten with a single "catchall" question [29]. Second, questions are asked using an "unfolding bracketing" method. Respondents who are unable or unwilling to provide an exact figure are presented with a series of questions such as "Was it more (or less) than $\$ 2000$ ?". Based on the responses to these questions, an exact dollar figure is imputed [30]. The bracketing procedure has been shown to reduce underreporting of economic concepts such as OOP spending [31]. Another indication of the validity of the HRS OOP spending information is that OOP spending increases with utilization of health care (within insurance coverage categories), suggesting that the HRS spending data are internally consistent [32].

A second issue concerns which HRS wave should be used for information on OOP spending. We use the HRS interview after ADAMS because it has the most relevant information on spending and utilization occurring during the assessment. This is because the ADAMS was conducted in the middle of the reference period for the OOP spending questions from the HRS interview after ADAMS. This can be seen in the study diagram depicted in Figure 1, which shows the reference period for the HRS questions on OOP spending and the approximate timing of the ADAMS diagnoses. The drawback of this approach is that ADAMS subjects who did not complete the subsequent HRS

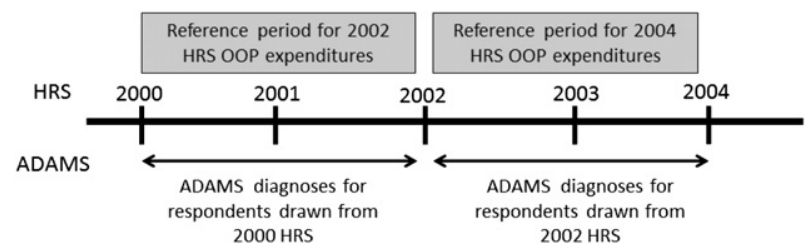

Fig. 1. Reference period for the questions on out-of-pocket spending and the timing of the Aging, Demographics, and Memory Study diagnoses. 
interview cannot be used in the analysis. In the discussion section, we describe the potential implications of excluding these individuals and discuss the results of a sensitivity analysis where we use information drawn from the HRS interview used to select individuals into ADAMS.

\subsection{Utilization of other types of care}

To investigate the hypothesis that dementia reduces spending on two types of health care services, which are usually only partially covered by insurance, we examine the association between dementia and the use of prescription medications and dental care. Both of them receive much less generous coverage by Medicare (at least before Medicare Part D, which was adopted after the study period) than other services. The first outcome we study is a variable indicating whether respondents had taken less medication than what was prescribed because of the medication's cost. This measure is especially useful for our purposes because it relates specifically to foregone care due to an inability to pay, and it has been used in other studies examining whether patients underutilize medications owing to cost $[33,34]$. The second measure is whether the HRS respondent had visited the dentist in the past 2 years. This outcome is somewhat less useful because individuals with dementia might be unable to visit the dentist because of their cognitive impairment [21] rather than because they cannot pay for it.

\subsection{Controls for comorbidities and demographic confounders}

Because dementia may be correlated with other determinants of OOP spending, it is important to adjust for factors that could confound the relationship between dementia and OOP spending. Fortunately, the HRS has considerable information on demographic and health conditions that we control for in the analysis of OOP spending. In the analysis, we used the following measures of demographic and economic status as controls for potential confounders: age (and age squared), proxy respondent status, marital status, number of children (dichotomous variables for zero or one child, two children, and three or more children), race (a dichotomous variable for whether the respondent is white), health insurance status (dichotomous variables for covered by long-term care insurance, covered by Medicaid, and covered by supplemental private insurance), gender, education (dichotomous variables for high school dropout, high school graduate, and at least some college), and household income. We also used information on the following comorbidities (all defined as dichotomous variables): stroke, diabetes, heart disease, hypertension, lung disease, cancer, psychiatric problems (which includes depression), and arthritis.

\section{Methods}

Our goal is to estimate the increase in OOP spending attributable to dementia. The simplest estimates we report simply compare the average spending levels by dementia status. These unadjusted estimates are useful for describing patterns in the data but may be misleading for understanding the increase in OOP spending attributable to dementia.

Therefore, our preferred approach is to estimate multivariate models that control for potential confounding factors. For the OOP spending measures, we estimate ordinary least squares regression models. Estimates from this type of model are informative about how average OOP spending changes with dementia status, conditional on covariates included in the model. For dichotomous outcomes, we use logistic regression.

The multivariate models include controls for all the demographic, economic, and comorbidity measures listed previously. These variables were chosen because they may be associated with dementia but may themselves have independent effects on OOP spending or health care utilization. For instance, we control for marital status and the number of offspring a respondent has because both of these are correlated with dementia status in the data but may affect the demand for nursing home care, as care by relatives can be a substitute for institutional care [35]. We did not control for self-reported health or functional status limitations because these are likely to be manifestations of dementia, and therefore, any costs associated with them should be attributed to dementia.

As noted in section 2.2., the dementia status measure categorizes individuals into one of three categories-normal cognitive functioning, CIND, and demented. To handle this three-level classification scheme, the statistical models include two dummy variables - the first equal to one if an individual has dementia and zero otherwise, and the second equal to one if an individual is CIND and zero otherwise. Estimates of the average level of OOP spending for each dementia category are then obtained using predictions from the ordinary least squares regressions when the covariates (other than the dementia status indicators) are set to the overall sample mean. Similarly, we compute the incidence rate for dichotomous outcomes (e.g., foregoing prescription medication owing to cost) by dementia status level using predictions from the logistic regression.

Comparing predicted average spending (or the incidence rate for a dichotomous outcome) between different dementia levels has the interpretation of the difference in the average of a given outcome associated with a change from one dementia status category to another after adjusting for the covariates and comorbidities listed previously. We report the $P$ value corresponding to the hypothesis test that adjusted average spending (or the incidence rate) is the same across the three dementia levels. We also discuss whether the incremental change in a given outcome associated with a change from normal cognitive functioning to CIND is the same as the change associated with progressing from CIND to dementia, as this is informative about the trend in an outcome associated with a decline in cognitive functioning. 
All analyses use the ADAMS sample weights that account for nonproportional sampling and differential nonresponse. Furthermore, standard errors are computed using STATA 10's "survey" commands that adjust for the complex survey design of ADAMS (StataCorp LP, College Station, TX).

\section{Results}

\subsection{Descriptive statistics}

Table 1 shows descriptive statistics of the analysis sample. Relative to sample members who were diagnosed as "normal," those with dementia are 6.3 years older $(P<.01)$, more likely to be nonwhite $(P=.02)$, single $(P<.01)$, and to have a proxy interview $(P<.01)$. Individuals with dementia also have lower socioeconomic status, as indicated by the higher incidence of having less than a high school degree $(P<.01)$ and lower average household income $(P<.01)$. There are also differences by dementia status in the likelihood of having certain comorbidities, such as stroke and heart disease, although other conditions, such as hypertension, diabetes, lung diseases, and cancer, show smaller and statistically insignificant differences across dementia diagnoses. With a few exceptions (such as the fraction of male subjects), the average of these characteristics among those with CIND falls in between the average for individuals diagnosed as normal or having dementia.

\subsection{Unadjusted comparisons by dementia status}

Unadjusted OOP spending and health care utilization by dementia status are reported in Table 2 . The results clearly show that OOP spending is strongly associated with having dementia. Average OOP spending among those with dementia is $\$ 10,039$, which is more than four times the average for individuals diagnosed as normal, and twice the mean for those classified as CIND $(P<.01)$.

These differences are concentrated in the right tail of the distribution of OOP spending. The incidence of having annual spending exceeding $\$ 5000$ is $28 \%$ among those with dementia compared with only $11 \%$ among those diagnosed as normal $(P<.01)$. Virtually all of these differences are driven by higher OOP spending on nursing home care by those with dementia. Nearly $30 \%$ of demented individuals have some OOP spending on nursing home care compared with only $1 \%$ of those classified as normal $(P<.01)$, and individuals with dementia spend an average of $\$ 7175$ per year on nursing home care compared with $\$ 1529$ for those classified as CIND and close to zero for those with normal cognitive functioning $(P<.01)$. Notably, the increase in OOP spending on nursing homes associated with a change from CIND to dementia (\$5646) is nearly four times the increase associated with a change from normal cognitive functioning to CIND ( $\$ 1525 ; P=.03$ ), suggesting that the trend in OOP spending on nursing homes associated with cognitive decline accelerates as individuals become demented. In contrast, spending on prescription drugs and

Table 1

Descriptive statistics by dementia status

\begin{tabular}{|c|c|c|c|c|c|}
\hline & All & Demented & CIND & Normal & $P$ value \\
\hline Male & 0.390 & 0.311 & 0.456 & 0.382 & .055 \\
\hline Married & 0.449 & 0.160 & 0.392 & 0.515 & $<.001$ \\
\hline One or no children & 0.243 & 0.334 & 0.293 & 0.212 & .040 \\
\hline Two children & 0.204 & 0.189 & 0.146 & 0.225 & .140 \\
\hline White & 0.858 & 0.777 & 0.800 & 0.889 & .017 \\
\hline Hispanic & 0.050 & 0.044 & 0.057 & 0.049 & .709 \\
\hline Have long-term care insurance & 0.104 & 0.051 & 0.114 & 0.110 & .179 \\
\hline Have supplemental private insurance & 0.306 & 0.198 & 0.297 & 0.327 & .020 \\
\hline Covered by Medicaid & 0.114 & 0.316 & 0.151 & 0.068 & $<.001$ \\
\hline Less than high school & 0.345 & 0.540 & 0.452 & 0.279 & $<.001$ \\
\hline High school graduate & 0.271 & 0.202 & 0.286 & 0.278 & .113 \\
\hline More than high school & 0.384 & 0.258 & 0.263 & 0.444 & $<.001$ \\
\hline Months between ADAMS and after HRS interview & 10.3 & 10.2 & 10.4 & 10.3 & .976 \\
\hline Age & 80.0 & 84.9 & 81.9 & 78.6 & $<.001$ \\
\hline Household income & 33,608 & 20,166 & 22,877 & 39,299 & $<.001$ \\
\hline Stroke & 0.153 & 0.459 & 0.214 & 0.082 & $<.001$ \\
\hline Diabetes & 0.209 & 0.188 & 0.278 & 0.191 & .157 \\
\hline Heart disease & 0.357 & 0.455 & 0.519 & 0.288 & $<.001$ \\
\hline Hypertension & 0.638 & 0.613 & 0.589 & 0.657 & .513 \\
\hline Lung disease & 0.106 & 0.111 & 0.188 & 0.079 & .079 \\
\hline Cancer & 0.206 & 0.146 & 0.231 & 0.208 & .194 \\
\hline Psychological condition & 0.195 & 0.353 & 0.183 & 0.172 & $<.001$ \\
\hline Arthritis & 0.761 & 0.783 & 0.777 & 0.752 & .733 \\
\hline Proxy interview & 0.095 & 0.452 & 0.134 & 0.022 & $<.001$ \\
\hline Number of observations & 743 & 229 & 215 & 299 & \\
\hline
\end{tabular}

Abbreviations: CIND, cognitive impairment, not demented; HRS, Health and Retirement Study; ADAMS, Aging, Demographics, and Memory Study. NOTE. $P$ value refers to differences in means across dementia status categories. 
Table 2

Unadjusted annual OOP spending and health care utilization, by dementia status

\begin{tabular}{|c|c|c|c|c|c|c|c|c|c|}
\hline & \multicolumn{2}{|c|}{ All $(\mathrm{N}=743)$} & \multicolumn{2}{|c|}{ Demented $(\mathrm{N}=229)$} & \multicolumn{2}{|c|}{ CIND $(\mathrm{N}=215)$} & \multicolumn{2}{|c|}{ Normal $(\mathrm{N}=299)$} & \multirow[b]{2}{*}{$P$ value } \\
\hline & Mean & SE & Mean & SE & Mean & SE & Mean & SE & \\
\hline Total annual OOP spending & 3705.2 & 417.4 & $10,039.2$ & 2336.5 & 5061.8 & 520.1 & 2200.8 & 216.7 & $<.001$ \\
\hline Annual OOP spending on nursing home care & 1146.9 & 384.2 & 7175.1 & 2259.6 & 1528.8 & 962.5 & 4.3 & 3.6 & .009 \\
\hline Annual OOP spending on prescription drugs & 1567.3 & 159.7 & 1425.6 & 193.3 & 1893.1 & 406.0 & 1487.7 & 165.4 & .525 \\
\hline Annual OOP spending on other care & 991.0 & 199.6 & 1438.5 & 438.0 & 1639.9 & 848.9 & 708.8 & 119.0 & .518 \\
\hline Annual OOP spending on nursing home care $>0$ & 0.057 & 0.011 & 0.289 & 0.057 & 0.083 & 0.027 & 0.010 & 0.006 & $<.001$ \\
\hline Annual OOP spending $>\$ 5000$ & 0.147 & 0.019 & 0.282 & 0.053 & 0.185 & 0.038 & 0.112 & 0.018 & $<.001$ \\
\hline Did not take prescribed medications owing to cost & 0.054 & 0.012 & 0.036 & 0.015 & 0.053 & 0.022 & 0.057 & 0.017 & .746 \\
\hline Visited dentist in past 2 years & 0.583 & 0.028 & 0.367 & 0.043 & 0.499 & 0.056 & 0.645 & 0.037 & $<.001$ \\
\hline
\end{tabular}

Abbreviations: OOP, out of pocket; SE, standard error.

NOTE. $P$ value refers to differences across dementia status categories. Sample size for estimates of the fraction not taking drugs owing to cost is 739 , and sample size for dentist visits in previous 2 years is 740 .

all other types of medical care does not vary significantly across dementia categories.

Despite much higher OOP spending for health care among individuals with dementia, the likelihood of not taking prescribed medication is not significantly related to dementia status. However, only one-third of individuals with dementia reported visiting a dentist in the previous 2 years, compared with two-thirds of individuals classified as normal $(P<.01)$.

\subsection{Multivariate analyses}

Table 3 shows the relationship between dementia status and OOP spending and health care utilization after controlling for demographic characteristics and comorbidities (see Tables A1 and A2 for the complete regression output). The unadjusted differences in OOP spending remain intact after controlling for potential confounders. In particular, the adjusted differences in total annual OOP spending indicate significant differences across dementia categories $(P$ $<$.01) that are very similar to the unadjusted figures; for example, the adjusted difference in OOP spending between those classified as normal and demented is $\$ 5646$, which is only somewhat less than the unadjusted difference of $\$ 7838$. Furthermore, these differences are driven by higher OOP spending on nursing home care among patients with dementia $(P<.01)$, and by more than a fourfold increase in the likelihood of reporting OOP spending greater than $\$ 5000(P<.01)$. The results also suggest that the increase in OOP spending associated with cognitive decline accelerates with the onset of dementia. For example, after adjusting for covariates, the increase in OOP spending on nursing home care associated with a change from normal cognitive functioning to CIND is statistically insignificant and only $\$ 484(P=.41)$, whereas the increase associated with a change from CIND to dementia is $\$ 2638(P=.04)$.

Despite the sizable OOP spending burden associated with dementia, however, there is no evidence that dementia leads to a reduction in other types of care that are often paid for OOP. Dementia is not significantly related to the probability of foregoing prescription medications owing to cost $(P=.34)$. Similarly, the adjusted differences in the use of dental care are smaller and no longer statistically significant after controlling for potential confounders $(P=.76)$.

\subsection{Sensitivity analyses}

One concern with the way the models are estimated has to do with the way they control for proxy respondent status. As

Table 3

Adjusted annual OOP spending and health care utilization by dementia status

\begin{tabular}{|c|c|c|c|c|c|c|c|}
\hline & \multicolumn{2}{|l|}{ Demented } & \multicolumn{2}{|l|}{ CIND } & \multicolumn{2}{|l|}{ Normal } & $P$ value \\
\hline Total annual OOP spending & 8216.3 & 1460.7 & 4872.1 & 531.7 & 2569.9 & 281.8 & $<.001$ \\
\hline Annual OOP spending on prescription drugs & 1995.7 & 346.3 & 2035.2 & 446.8 & 1345.9 & 145.9 & .261 \\
\hline Annual OOP spending on other care & 1108.3 & 562.9 & 1652.6 & 787.9 & 760.7 & 128.6 & .364 \\
\hline Annual OOP spending $>\$ 5000$ & 0.308 & 0.384 & 0.165 & 0.360 & 0.073 & 0.212 & $<.001$ \\
\hline
\end{tabular}

NOTE. Entries are the predicted average of the outcome variable evaluated for a given dementia category at the overall sample mean for all covariates in the model, except for the dementia status indicator variables. Models adjust for proxy respondent status, marital status, number of children (one, two, or more than two), race, insurance status (long-term care insurance, Medicaid, supplemental private insurance), gender, education, age and age squared, and chronic conditions (stroke, diabetes, heart disease, hypertension, lung disease, cancer, psychiatric problems, and arthritis). $P$ value refers to differences across dementia status categories. Sample size for estimates of the fraction not taking drugs owing to cost is 739 , and sample size for dentist visits in previous 2 years is 740 . 
proxy status is frequently a manifestation of cognitive impairment, controlling for proxy status could lead to understated estimates of the relationship between dementia and OOP spending. The results obtained when not controlling for proxy status are consistent with the patterns described here, although, as expected, the magnitudes of the estimates are somewhat larger. For instance, the increase in total OOP spending associated with dementia (relative to normal) is $\$ 7674$ without controlling for proxy status compared with $\$ 5646$ when adjusting for proxy status.

We also examined the sensitivity of the cutoff used to identify very large OOP expenditures. The results indicate that dementia is associated with a significant increase in the likelihood of incurring large annual OOP expenditures, where the cutoff used to define "large" ranges from $\$ 4000$ to $\$ 10,000$.

\section{Discussion}

This article estimates the magnitude of the OOP spending burden associated with dementia. The findings indicate that dementia is associated with higher levels of average OOP spending and that this association is driven mainly by spending on nursing home care. In year 2010 prices, our best estimate is that those with dementia (or their families) spend OOP $\$ 5646$ per year more than those who have normal cognitive functioning. To put this in perspective, according to tabulations we did using the Consumer Expenditure Survey, average spending in 2009 by households where the head of the household was 75 years or older was $\$ 21,900$.

Because the ADAMS sample is nationally representative of the population over the age of 70 years, we can estimate the total OOP spending associated with dementia. To bring this up to date, we use the total population over the age of 70 years in 2009, which we take from Census estimates to be 28.1 million [36]. We take dementia prevalence to be $13.9 \%$ (as estimated in the full ADAMS sample) and multiply it by the excess of spending associated with dementia (\$5646). From these data, we estimate that there is $\$ 22.0$ billion of additional OOP spending in 2010 that is associated with dementia-related care.

Despite the strong relationship between very high levels of OOP spending and dementia, there is no evidence of a relationship between dementia and the use of other care that is typically paid for OOP. Specifically, dementia is not significantly related to the utilization of dental care or the likelihood of foregoing prescribed medication owing to cost. We interpret this lack of a relationship to mean that OOP spending for dementia does not drive out spending for these health care services and goods.

The major strengths of this study are twofold. One is that it is the first to examine the link between dementia and health care spending using nationally representative data. Second, it is also the first study that we know of to provide evidence about whether dementia is associated with a reduction in the use of two types of medical care that are frequently financed, at least in part, through OOP spending. This contribution is important because a key concern about high OOP spending among elderly individuals is that it will reduce the use of important medications and other forms of care [2,33]. The findings reported here suggest that even large OOP spending burdens might not lead to reduced health care utilization among elderly individuals with dementia.

However, there are several limitations of this analysis that should be considered when evaluating these findings. First, this is an observational study and part of the association between dementia and OOP spending may be due to unobserved confounds. That said, the fact that the effects on OOP spending arise almost entirely through an effect on nursing home care suggests bias from omitted variables may be small; if the observed relationship was purely due to worse health generally among those with dementia, then we would expect to see higher spending on medical care more broadly and not just on long-term care services. The fact that this pattern is not observed suggests that the estimates we obtain may reflect differences in spending attributable to dementia.

Second, the study relies on self-reported information on OOP spending and utilization of care, and we have no way of directly assessing the validity of these outcomes. However, studies [4] using data from the Medicare Current Beneficiary Survey that include information from Medicare claims records that are used to cross-check self-reported OOP spending also show that OOP spending is higher among patients with chronic conditions. Although the lack of Medicare coverage for long-term care makes these findings not fully comparable with those reported here, it is important to note that the HRS questions are designed to minimize underreporting bias in OOP spending measures [31], and other research [32] supports the internal consistency of the HRS OOP spending measures. Similarly, the validity of self-reported data on dental care utilization has been found to be high [37], and several studies have found that self-reported information on prescription drug use corresponds well with information from claims data [38-40].

However, we acknowledge that the OOP spending measures we use likely contain some misreporting, and this represents an important limitation of the study. In particular, this misreporting could result in bias for our estimates of the association between dementia and spending if the severity and direction of misreporting differ by dementia status. However, we see no obvious reason why the quality of the spending information would differ between individuals with and without dementia to such a degree that it would fully account for the very large OOP spending burden of dementia we find.

This claim finds further support in auxiliary analyses (available on request) conducted with Medicare claims data linked to the ADAMS-HRS data. The results from these analyses indicate that dementia is associated with large increases in spending on nursing home care but is not associated with increases in spending on other types of 
care. Medicare only covers a small subset of total nursing home stays (specifically those for postacute care); therefore, the magnitude of the association between dementia and nursing home spending in the Medicare claims data is not directly comparable with the association between dementia and OOP spending (which is driven precisely by those nursing home stays not covered by Medicare). Nonetheless, in both the claims records and in the self-reported OOP spending data, we find that dementia is significantly related to expenditures on nursing home care but not on other types of care. This similarity provides reassurance that differential misreporting is unlikely to be the chief explanation for our main findings.

Third, the sample used in this article excludes ADAMS participants who did not complete the HRS interview after ADAMS. Consequently, our sample has better cognitive health, as evidenced by the lower dementia prevalence (11.4\%) compared with the entire ADAMS sample (13.9\%). Because our sample is healthier relative to the population, some of the more costly cases of dementia may be excluded. Thus, our estimates may understate the OOP spending burden of dementia.

We also explored using spending and utilization from the HRS wave before ADAMS rather than from the HRS wave after ADAMS. Many of the findings were similar to those reported previously, including significantly higher levels of spending on nursing home and hospital care, much higher incidence of very large OOP expenditures among those with dementia, and no significant differences in the utilization of dental care or prescription drugs. However, we found two differences. Among the respondents interviewed in both waves $(\mathrm{N}=458)$, spending among those diagnosed with dementia in ADAMS was substantially lower in the HRS wave preceding ADAMS than in the wave after it $\$ 6009$ compared with $\$ 10,039, P=.09$ ). This suggests that some of these individuals had not yet progressed to dementia status in the previous HRS wave. Second, when we adjusted for covariates, total OOP spending did not differ significantly across dementia diagnosis categories in the previous HRS wave. Qualitatively this is to be expected because of classification error-some individuals classified as demented in ADAMS would not have yet been demented in the previous HRS waves. Such misclassification at the time of the cost measurement (in the previous HRS wave) would attenuate the relationship between dementia and cost. We interpret these differences as support for using cost measurement from the HRS wave after the ADAMS assessment rather than from the previous HRS wave.

A fourth limitation concerns the generalizability of the finding that dementia is not associated with reductions in other care and health goods that are frequently paid for OOP. One reason is that the costs of prescription medications may be included in nursing home fees. In auxiliary analyses, we find mixed support for this hypothesis; nursing home residence is associated with a reduction in the likelihood of reporting positive OOP spending on prescription drugs
$(P<.01)$, whereas no statistically significant relationship was found between OOP spending for nursing homes and for prescription medications. A second reason why these results may not be generalizable is that physicians typically reduce the intensity of treatment for other conditions as a patient's dementia progresses, thereby mitigating the tradeoff between paying for care related to dementia and other forms of medical care. In contrast, such a tradeoff might be more pressing for patients experiencing high OOP expenditures for treatment of different chronic conditions.

Finally, this analysis does not address other ways in which high OOP spending could diminish the wellbeing of patients with dementia. For instance, large OOP expenditures could make it difficult for individuals to afford other necessities such as nutritious food, clothing, and heat or air conditioning. However, the OOP expenses observed in our data appear to be for nursing home care, which is where these needs are presumably met. A related point is that the OOP spending burden may be borne, at least to some extent, by the children of individuals with dementia in the form of smaller or nonexistent bequests. Future research should determine who bears the burden of the large OOP expenses associated with dementia.

\section{Acknowledgments}

Financial support from the National Institute on Aging under grant R01AG030155 is gratefully acknowledged. The HRS and ADAMS are funded by the National Institute on Aging (U01 AG009740), and administered by the Institute for Social Research at the University of Michigan.

\section{References}

[1] Altman B, Cooper PF, Cunningham PJ. The case of disability in the family: impact on health care utilization and expenditures for nondisabled members. Milbank Q 1999;77:39-75.

[2] Federman AD, Adams AS, Ross-Degman D. Supplemental insurance and use of effective cardiovascular drugs among elderly Medicare beneficiaries with coronary heart disease. J Am Med Assoc 2001; 276:1473-9.

[3] Cubanski J, Damico A, Neuman T. Health care on a budget: an analysis of spending by Medicare households. The Henry J. Kaiser Family Foundation, 2009. Cited January 6, 2011. Available at: http://www. kff.org/medicare/upload/7859.pdf.

[4] Gross D, Brangan N. Out of pocket spending on health care by Medicare beneficiaries age 65 and older: 1999 projections. Washington, D.C: Public Policy Institute Issue Brief, American Association of Retired Persons; 1999. p. 1-14.

[5] Himmelstein DU, Warren E, Thorne D, Woolhandler S. Illness and injury as contributors to bankruptcy. Health Aff 2006;25:74-83.

[6] Neuman T, Cubanski J, Damico A. Revisiting "skin in the game" among Medicare beneficiaries. an updated analysis of the increasing financial burden of health care spending from 1997 to 2005, 2009. Cited January 6, 2011. Available at: http://www.kff.org/medicare/ upload/7860.pdf.

[7] Schneeweiss S, Patrick A, Pedan A, Varasteh L, Levin R, Liu N, Shrank W. The effect of Medicare part D coverage on drug use and cost sharing among seniors without prior drug benefits. Health Aff 2009;28:w305-16. 
[8] Cassel CK, Besdine RW, Siegel LC. Restructuring Medicare for the next century: what will beneficiaries really need? Health Aff 1999; 18:118-31.

[9] Smith GE, O'Brien PC, Ivnik RJ. Prospective analysis of risk factors for nursing home placement of dementia patients. Neurology 2001; 57:1467-73.

[10] Yaffe K, Fox P, Newcomer R. Patient and caregiver characteristics and nursing home placement in patients with dementia. J Am Med Assoc 2002;287:2090-7.

[11] Newcomer R, Clay R, Luxenberg J. Misclassification and selection bias when identifying Alzheimer's disease solely from Medicare claims records. J Am Geriatr Soc 1999;47:215-21.

[12] O'Brien E. Medicare and long-term care. Georgetown University Long-Term Care Financing Project; 2007. Cited January 6, 2011. Available at: http://ltc.georgetown.edu/pdfs/medicare0207.pdf.

[13] Gardner L, Gilleskie D. The effects of state Medicaid policies on the dynamic savings patterns of the elderly. Cambridge, MA: NBER; 2006. NBER Working Paper \#12208.

[14] Norton E. Elderly assets, Medicaid policy, and spend-down in nursing homes. Rev Income Wealth 2005;41:309-29.

[15] Spence D, Weiner J. Estimating the extent of Medicaid spend-down in nursing homes. J Health Polit Policy Law 1990;15:607-26.

[16] Brummel-Smith K, Weiner M, Powe N. Alzheimer's disease under managed care: implications from Medicare utilization and expenditure patterns. J Am Geriatr Soc 1998;46:762-71.

[17] Gutterman E, Markowitz A, Lewis B. Cost of Alzheimer's disease and related dementia in managed-Medicare. J Am Geriatr Soc 1999; 47:1065-73.

[18] Hill J, Futterman R, Duttagupta S. Alzheimer's disease and related dementias increase costs of comorbidities in managed Medicare. Neurology 2002;58:62-70.

[19] Langa KM, Larson E, Wallace RB, Fendrick AM, Foster NL, Kabeto MU, Weir DR, Willis RJ, Herzog AR. Out of pocket health care expenditures among older Americans with dementia. Alzheimers Dis Assoc Disord 2004;18:90-8.

[20] McCormick WC, Hardy J, Kukull WA, Bowen JD, Teri L, Zitzer S, Larson E. Healthcare utilization in costs in managed care patients with Alzheimer's disease during the last few years of life. J Am Geriatr Soc 2001;49:1156-60.

[21] Walsh G, Wu B, Mitchell J, Berkman L. Cognitive function and acute care utilization. J Gerontol Br 2003;58:S38-49.

[22] Andersen CK, Sogaard J, Hansen E, Kragh-Sorensen A, Hastrup L. The cost of dementia in Denmark: the odense study. Dement Geriatr Cogn Disord 1997;10:295-304.

[23] Di Carlo A, Baldereschi M, Amaducci L, Maggi S, Grigoletto F, Scarlato G. Cognitive impairment without dementia in older people: prevalence, vascular risk factors, impact on disability: the Italian longitudinal study on aging. J Am Geriatr Soc 2000;48:775-82.

[24] Callahan CM, Hendrie HC, Tierney WM. Documentation and evaluation of cognitive impairment in elderly primary care patients. Ann Intern Med 1995;122:422-9.
[25] Juster F, Suzman R. An overview of the health and retirement study. J Hum Resour 1995;30:S7-56.

[26] Heeringa SG, Fisher G, Hurd M, Langa KM, Ofstedal MB, Plassman BL. Aging, demographics and memory study (ADAMS). Sample design, weighting and analysis for ADAMS. University of Michigan; 2009. Cited January 6, 2011. Available at: http://hrsonline.isr.umich edu/sitedocs/userg/ADAMSSampleWeights_Jun2009.pdf.

[27] Langa KM, Plassman BL, Wallace RB, Herzog AR, Heeringa S. The aging, demographics and memory study: study design and methods. Neuroepidemiology 2005;25:181-91.

[28] Cohen S, Carlson B. A comparison of household and medical provider reported expenditures in the 1987 NMES. J Off Stat 1994;10:3-29.

[29] Bound J, Brown C, Mathiowetz N. Measurement error in survey data. In: Heckman J, Leamer E, eds. Handbook of econometrics, Vol. 5. North-Holland: Elsevier Science; 2001. p. 3705-842.

[30] Cao H. Impute: a SAS application system for missing value imputations. Ann Arbor, MI: University of Michigan; 2009. Cited January 6, 2011. Available at: http://hrsonline.isr.umich.edu/sitedocs/userg/ dr-007.pdf

[31] Hurd M, Rodgers W. The effects of bracketing and anchoring on measurement in the health and retirement study. Ann Arbor, MI: Institute for Social Research; 2008.

[32] Johnson RW, Crystal S. Uninsured status and out-of-pocket costs at midlife. Health Serv Res 2000;35(5 Pt 1):911-32.

[33] Steinman M, Sands L, Covinsky K. Self-restriction of medications due to cost in seniors without prescription drug coverage. J Gen Intern Med 2001;16:793-9.

[34] Heisler M, Choi H, Rosen A, Vijan S, Kabeto M, Langa K, Piette D. Hospitalizations and deaths among adults with cardiovascular disease who underuse medications because of cost: a longitudinal analysis. Med Care 2010;48:87-94.

[35] Langa K, Chernew M, Kabeto MU, Herzog R, Ofstedal MB, Willis R, Wallace R, Mucha L, Straus W, Fendrick M. National estimates of the quantity and cost of informal caregiving for the elderly with dementia. J Gen Intern Med 2001;16:770-8.

[36] U.S. Census Bureau. US Interim Projections 2000-2050. Available at: http://www.census.gov/population/www/projections/usinterimproj/ usproj2000-2050.xls. Accessed December 8, 2010.

[37] Gilbert GH, Rose JS, Shelton BJ. A prospective study of the validity of data on self-reported dental visits. Community Dent Oral Epidemiol 2002;30:352-62.

[38] Sjahid SI, van der Linden PD, Stricker BH. Agreement between the pharmacy medication history and patient interview for cardiovascular drugs: the Rotterdam elderly study. Br J Clin Pharmacol 1998;45:591-5.

[39] Rost K, Roter D. Predictors of recall of medication regimens and recommendations for lifestyle change in elderly patients. Gerontologist 1987;27:510-5.

[40] West SL, Savitz DA, Koch G, Strom BL, Guess HA, Hartzema A. Recall accuracy for prescription medications: self-report compared with database information. Am J Epidemiol 1995;142:1103-12. 
Table A1

Regression coefficients for annual OOP spending levels

\begin{tabular}{|c|c|c|c|c|}
\hline Dependent variable & Total OOP spending & $\begin{array}{l}\text { OOP spending for } \\
\text { nursing home care }\end{array}$ & $\begin{array}{l}\text { OOP spending for } \\
\text { prescription drugs }\end{array}$ & $\begin{array}{l}\text { OOP spending for } \\
\text { other care }\end{array}$ \\
\hline CIND & $2302.21 *(612.31)$ & $721.06(872.31)$ & $689.26(490.88)$ & $891.89(797.58)$ \\
\hline Demented & $5646.42 *(1412.87)$ & $4649.01 * *(1307.83)$ & $649.74(396.16)$ & $347.68(630.57)$ \\
\hline Male & $-462.41(1289.07)$ & $292.62(747.73)$ & $34.30(270.83)$ & $-789.33(526.86)$ \\
\hline Married & $-1063.19(1071.96)$ & $-1105.86 * * *(499.46)$ & $-140.46(385.16)$ & $183.13(419.72)$ \\
\hline One or no children & $-30.35(857.12)$ & $525.97(867.52)$ & $-23.66(245.49)$ & $-532.66(447.37)$ \\
\hline Two children & $200.69(542.28)$ & $637.81(503.74)$ & $-116.06(242.06)$ & $-321.06(341.39)$ \\
\hline White & $901.23(733.60)$ & $308.00(595.01)$ & $696.56 * * *(332.94)$ & $-103.32(428.36)$ \\
\hline Hispanic & $935.66(935.15)$ & $1144.43(639.40)$ & $363.44(232.74)$ & $-572.21(404.67)$ \\
\hline Have long-term care insurance & $-729.23(656.90)$ & $-785.10 * * *(374.54)$ & $249.65(462.05)$ & $-193.79(409.17)$ \\
\hline $\begin{array}{l}\text { Have supplemental private } \\
\text { insurance }\end{array}$ & $247.24(943.52)$ & $152.72(693.87)$ & $683.34 * * *(315.63)$ & $-588.81 * * *(256.64)$ \\
\hline Covered by Medicaid & $-3553.12 * *(1083.01)$ & $-1992.41(1018.89)$ & $-892.35^{* * * *}(335.12)$ & $-668.35 * * *(265.80)$ \\
\hline Less than high school & $-2291.15(1365.45)$ & $-2305.23(1465.64)$ & $176.62(419.55)$ & $-162.55(397.68)$ \\
\hline High school graduate & $59.61(1397.64)$ & $-1484.68(1119.40)$ & 362.53 (334.27) & $1181.76 * * *(567.60)$ \\
\hline $\begin{array}{l}\text { Months between ADAMS } \\
\text { and after HRS interview }\end{array}$ & $-0.61(1.31)$ & $0.11(0.93)$ & $-0.64(0.58)$ & $-0.09(0.57)$ \\
\hline Age & $51.02(138.92)$ & $28.04(138.41)$ & $9.01(27.11)$ & $13.97(74.71)$ \\
\hline Age squared & $-0.02(0.07)$ & $-0.01(0.07)$ & $-0.01(0.01)$ & $-0.01(0.04)$ \\
\hline Household income & $0.02 * *(0.01)$ & $-0.00(0.00)$ & $0.01(0.01)$ & $0.02 * * *(0.01)$ \\
\hline Stroke & $1900.43(1953.81)$ & $2310.62(1419.01)$ & $-259.67(315.24)$ & $-150.53(525.71)$ \\
\hline Diabetes & $449.41(1232.16)$ & $-751.70(548.07)$ & $581.18(366.34)$ & $619.93(586.30)$ \\
\hline Heart disease & $2116.96 * * *(819.18)$ & $1054.46(810.37)$ & $386.69(240.16)$ & $675.80(362.78)$ \\
\hline Hypertension & $1789.41 * * *(725.94)$ & $887.11(791.48)$ & $495.88(261.60)$ & $406.42(341.71)$ \\
\hline Lung disease & $1498.68(2004.37)$ & $2280.00(1941.78)$ & $-364.79(421.63)$ & $-416.54(608.94)$ \\
\hline Cancer & $339.16(1249.56)$ & $-202.57(708.55)$ & $-88.79(295.22)$ & $630.51(637.79)$ \\
\hline Psychological condition & $305.15(696.28)$ & $425.20(685.58)$ & $362.10(301.28)$ & $-482.15(480.86)$ \\
\hline Arthritis & $-517.01(552.76)$ & $-522.89(567.67)$ & $45.85(275.09)$ & $-39.97(289.62)$ \\
\hline Proxy interview & $5368.60(2723.88)$ & $3617.66(2215.17)$ & $-165.19(477.51)$ & $1916.13(2119.42)$ \\
\hline Intercept & $-28,156.65(67,474.10)$ & $-15,874.22(67,218.48)$ & $-3998.90(13,822.79)$ & $-8283.53(36,594.38)$ \\
\hline Number of observations & 743 & 743 & 743 & 743 \\
\hline
\end{tabular}

NOTE. Standard errors reported in parentheses.

$* P<.001$.

$* * P<.01$.

$* * * P<.05$. 
Table A2

Logistic regression coefficients for health care utilization and incidence of large OOP expenditures

\begin{tabular}{|c|c|c|c|}
\hline Dependent variable & Visited dentist & $\begin{array}{l}\text { Did not take prescription } \\
\text { medications owing to cost }\end{array}$ & $\begin{array}{l}\text { Annual OOP spending } \\
\text { greater than } \$ 5000\end{array}$ \\
\hline CIND & $-0.24(0.27)$ & $-0.25(0.59)$ & $0.92 *(0.35)$ \\
\hline Male & $-0.37(0.25)$ & $-1.27(0.79)$ & $0.32(0.35)$ \\
\hline Married & $0.64 *(0.29)$ & $0.56(0.54)$ & $-0.72(0.40)$ \\
\hline One or no children & $0.48(0.24)$ & $0.08(0.59)$ & $0.39(0.40)$ \\
\hline Two children & $0.40(0.23)$ & $-0.65(0.66)$ & $-0.24(0.23)$ \\
\hline Hispanic & $-0.86(0.63)$ & $-1.80 *(0.73)$ & $0.27(0.81)$ \\
\hline Have long-term care insurance & $0.74(0.45)$ & $0.97(0.80)$ & $-0.67(0.39)$ \\
\hline Have supplemental private insurance & $0.27(0.25)$ & $0.80(0.53)$ & $0.44(0.36)$ \\
\hline Covered by Medicaid & $-0.43(0.44)$ & $-1.25(0.78)$ & $-0.65(0.38)$ \\
\hline Less than high school & $-1.24 * * *(0.31)$ & $1.21 *(0.58)$ & $-0.42(0.43)$ \\
\hline High school graduate & $-0.62(0.32)$ & $0.83(0.87)$ & $0.53(0.33)$ \\
\hline Household income & $0.01 *(0.01)$ & $0.01(0.01)$ & $0.01 * * *(0.00)$ \\
\hline Stroke & $0.01(0.43)$ & $-0.01(0.99)$ & $-0.07(0.44)$ \\
\hline Diabetes & $0.36(0.25)$ & $0.15(0.55)$ & $0.65(0.53)$ \\
\hline Heart disease & $0.26(0.24)$ & $0.12(0.42)$ & $0.55^{*}(0.26)$ \\
\hline Hypertension & $-0.01(0.20)$ & $0.19(0.49)$ & $0.98 * *(0.34)$ \\
\hline Lung disease & $-0.25(0.43)$ & $0.66(0.88)$ & $-0.15(0.31)$ \\
\hline Cancer & $-0.29(0.31)$ & $0.74(0.53)$ & $-0.35(0.34)$ \\
\hline Psychological condition & $-0.40(0.32)$ & $2.02 * * *(0.52)$ & $0.03(0.35)$ \\
\hline Arthritis & $-0.01(0.36)$ & $0.56(0.78)$ & $0.25(0.32)$ \\
\hline Proxy interview & $0.27(0.36)$ & $-2.26^{*}(0.92)$ & $0.00(0.42)$ \\
\hline Intercept & $0.96(11.10)$ & $58.49 * *(20.73)$ & $4.39(16.13)$ \\
\hline Number of observations & 739 & 740 & 743 \\
\hline
\end{tabular}

NOTE. Standard errors reported in parentheses.

$* P<.05$.

$* * P<.01$.

$* * * P<.001$. 